\title{
A retrospective analysis of 29 isolated sphenoid fungus ball cases from a medical centre in Korea (1999-2012**
}

\author{
Tae Hoon Kim², Kyung Jin Na², Jun Ho Seok², Sung Jae Heo², Ji Hye Park², \\ Jung Soo Kim² \\ Rhinology 51: 280-286, 2013 \\ DOI:10.4193/Rhino12.145 \\ ' Department of Otorhinolaryngology-Head \& Neck Surgery, Daegu Fatima Hospital, Daegu, South Korea \\ *Received for publication: \\ ${ }^{2}$ Department of Otorhinolaryngology-Head \& Neck Surgery, School of Medicine, Kyungpook National University, \\ September 10,2012 \\ Daegu, South Korea \\ Accepted: February 8, 2013
}

\section{Summary}

Background: Isolated sphenoid sinus disease (ISSD) is rare. Fungus ball (FB) is the third most common ISSD. We analysed the characteristics of isolated sphenoid FB based on demographic data, presenting symptoms, preoperative computed tomography (CT), magnetic resonance imaging (MRI), and treatment outcomes.

Methodology: From 1999 to 2012, 29 patients were identified with isolated sphenoid FB. Demographic data; clinical characteristics; endoscopic, CT, and MRI findings and treatment outcomes were retrospectively analysed.

Results: The most common symptom was headaches, which were localized in various regions of the brain. Other symptoms were uncommon. The most common CT findings were sclerosis, calcification, enlarged sinus and total opacification. On T2-weighted MRI images, we most commonly observed signal void. Endoscopic transnasal paraseptal sphenoidotomy was performed in all patients, and for most, this was performed under local anaesthesia. No recurrence was observed in any patient.

Conclusion: Isolated sphenoid FB is predominantly observed in older women, and it is characterised by headaches and sclerosis of the sinus wall observed on CT scans. In cases of isolated sphenoid FB, endoscopic transnasal paraseptal sphenoidotomy can be successfully performed under local anaesthesia, which may facilitate rapid recovery and a low morbidity rate.

Key words: sphenoid sinus, Aspergillus, fungus ball

\section{Introduction}

Isolated sphenoid sinus disease (ISSD) is a rare disease because most sphenoid sinus lesions appear in conjunction with diseases of the posterior ethmoid sinuses. Recently, the detection rate of ISSD has increased because of the development of imaging techniques such as computed tomography (CT) and magnetic resonance imaging (MRI). Although the symptoms of ISSD are difficult to define in terms of precise characteristics, patients with this disease are known to most commonly complain of headache, visual disorder, and nasal obstruction ${ }^{(1-3)}$.

ISSD lesions involve sinusitis, fungal infection, mucocoeles, or tumours. Of these, fungal infection is the third most common cause of such lesions $(14.5 \%)^{(4)}$. Although fungus ball (FB) in 
the sphenoid sinus is rare, it is the most common disease of the paranasal sinuses. The most common causative fungus isolated from sphenoid FB, as well as from the paranasal sinuses, is Aspergillus fumigatus. The most frequently affected paranasal sinus is the maxillary, followed by the sphenoid, ethmoid, and frontal sinuses ${ }^{(5-11)}$. The symptoms of isolated sphenoid FB are nonspecific, as is the case for ISSD. Endoscopic examinations often do not indicate any abnormality. Therefore, both diagnosis and time to treatment are generally delayed.

The purpose of this study was to analyse the characteristics of isolated sphenoid FB based on presenting symptoms: preoperative endoscopy, CT, and MRI findings and treatment outcomes.

\section{Materials and methods}

\section{Patients}

Of all patients treated for FB between 1999 and 2012 at the Kyungpook National University Hospital in Daegu, only those with lesions confined to the sphenoid sinus were $(n=29)$ included in this study. We reviewed clinical records, including clinical presentation, radiological imaging, management, outcomes, histology, and follow-up. The Dehazo diagnostic criteria were used for diagnosing FB (Table 1) $)^{(12)}$.

\section{Clinical examination}

We performed endoscopic examination of all patients on their initial visit to our clinic. In patients with abnormal endoscopic findings or those who complained of persistent headache, computed tomography (CT) was performed (Figure 1). Some patients (17 of 29) underwent magnetic resonance imaging (MRI) because they had a neurological problem (e.g., visual disturbance) or erosion of the inner sinus wall on their CT scan (Figure 2).

\section{Surgery}

The surgery was performed under either local or general anaesthesia. If local anaesthesia was used, we administered moderate sedative drugs; $3 \mathrm{mg}$ of midazolam and $30 \mathrm{mg}$ of piroxicam potassium were administered 15 minutes before the surgery, and $50 \mathrm{mg}$ of meperidine hydrochloride was administered immediately before surgery. Vital signs were monitored during the surgery. The dosage of the sedative drugs administered was determined based on the patient's general condition.

Pledgets moistened with epinephrine were packed into the nasal cavity (between the nasal septum and the middle turbinate) for vasoconstriction, after which $2 \%$ lidocaine with 1:100,000 epinephrine was used for the local infiltration under $0^{\circ}$ scope. When sphenoidotomy was performed through the paraseptal approach, the superior turbinate was identified first and was partially resected to widen the sphenoethmoidal recess. After we identified the sphenoid ostium, using Lucae forceps or a suction tip we enlarged the natural ostium of the sphenoid to complete the sphenoidotomy. Finally, sinus irrigation with normal saline solution was performed to completely remove any fungal debris from the sphenoid sinus (Figure 3).

\section{Follow-up}

All patients were followed up by weekly endoscopy to remove crust and discharge during the first postoperative month. We recommended that patients perform nasal irrigation with saline solution 3 times a day for at least 1 month. After 1 month, they were followed up at 3 months, 6 months, and subsequently every year. We excluded patients who had allergic fungal rhinosinusitis, invasive fungal rhinosinusitis, or those who were immunocompromised.

\section{Results}

Twenty-nine patients ( $8 \%$ of a total of 362 diagnosed with FB) had isolated sphenoid FB. Of these, 10 (34.5\%) were men and 19 (65.5\%) were women, with a collective mean age of 60.4 years (range, 39-79 years). Various symptoms, including headache, posterior nasal drip, purulent rhinorrhea, nasal obstruction, and visual disturbance, were presented. The duration of symptoms ranged from 0 to 64 months (mean, 9.41 months). The most common symptom, presenting in 20 patients (69\%), was headache. Of these 20 patients, 7 experienced headaches in the frontal region (35\%), 5 in the parietal region (25\%), and 5 in the whole area (25\%). Two patients presented with headaches in the occipital region (10\%), and 1 in the vertex region (5\%). The other symptoms were posterior nasal drip (2 patients, 6.9\%), purulent rhinorrhea (1 patient, 3.4\%), nasal obstruction (2 patients, $6.9 \%$ ),

Table 1. Clinicopathological criteria for the diagnosis of a fungus ball (from Dehazo et al. ${ }^{(12)}$ ).

1. Radiologic evidence of sinus opacification with or without associated flocculent calcifications.

2. Mucopurulent, cheesy, or clay-like material within a sinus.

3. A matted, dense conglomeration of hyphae separate from, but adjacent to the sinus respiratory mucosa.

4. A chronic inflammatory response of variable intensity in the mucosa adjacent to fungal elements. This response includes lymphocytes, plasma cells, mast cells, and eosinophils without an eosinophil predominance or a granulomatous response. Allergic mucin is absent on haematoxylineosin-stained material.

5. No histologic evidence of fungal invasion of mucosa, associated blood vessels, or underlying bone visualised microscopically on Gomori methenamine silver or other stains specific for fungus. 

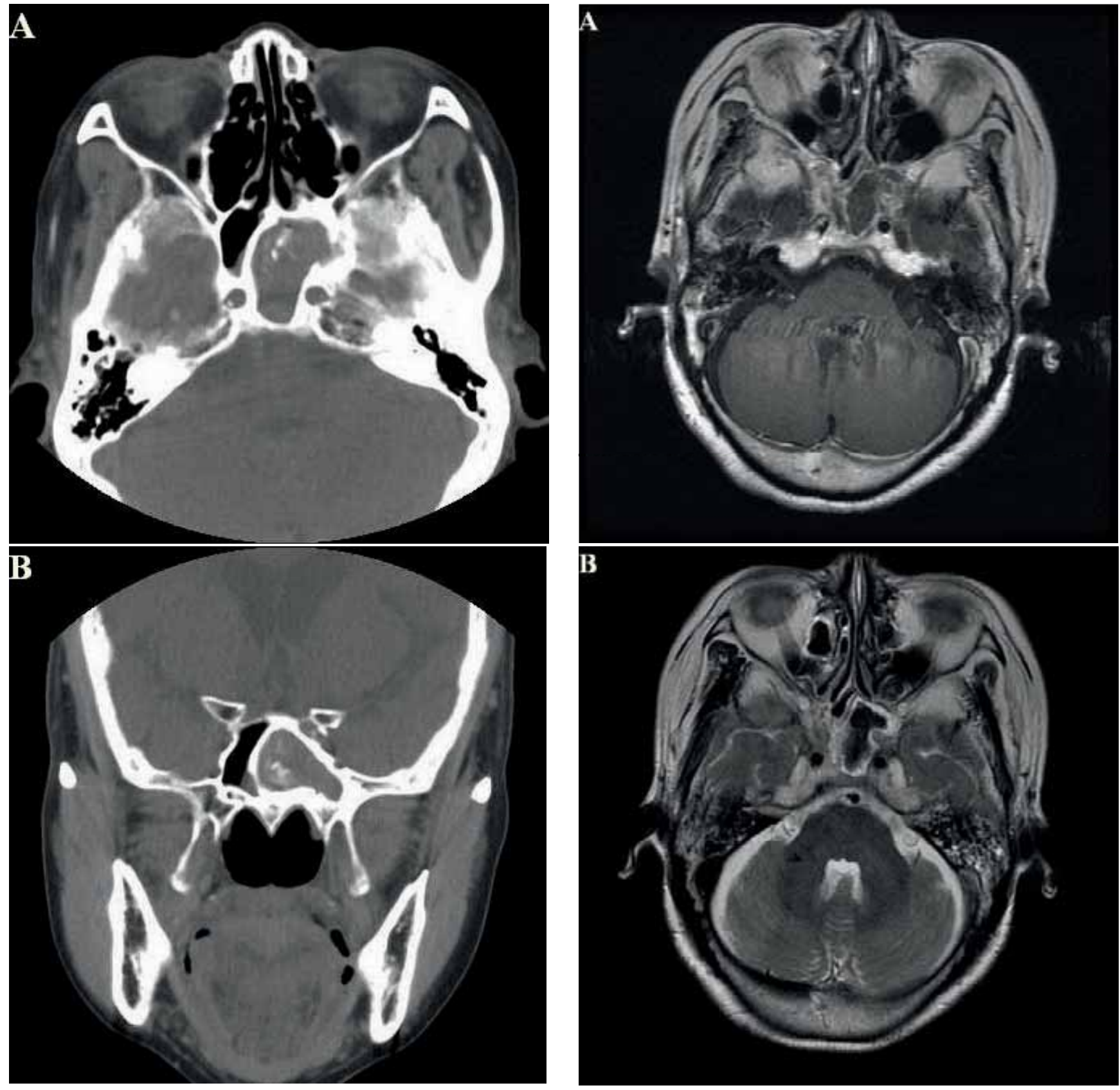

Figure 1. A computed tomography (CT) scan demonstrating total opacification of the sphenoid sinus on the left side with calcification and sclerosis of the sinus wall. A) Axial. B) Coronal.

Figure 2. Magnetic resonance imaging (MRI) scan demonstrating (A) isointensity on $\mathrm{T}-1$ weighted images with gadolinium and (B) signal void on T-2 weighted images.

and visual disturbance (1 patient, 3.4\%). Six (20.7\%) patients did not present any symptoms, and isolated sphenoid FB was detected incidentally during health screening. The endoscopic findings of 22 patients were normal; however, sphenoethmoidal recess polyps were observed in 4 (13.8\%) patients, mucopurulent discharge in $2(6.9 \%)$, and mucosal oedema of the sphenoethmoidal recess in 1 (3.4\%).

CT scans were performed preoperatively in all patients.
However, we could analyse the CT scans of only 23 patients because the scans of 6 patients were lost. Of the 23 patients for which CT scans were available, 22 had sclerosis of the sinus wall (95.6\%), 14 had an enlarged sinus (60.9\%), 14 had total opacification $(60.9 \%), 3$ had erosion of the inner sinus wall (13\%), and 16 had calcification (69.6\%). Seventeen patients underwent MRI (58.6\%). On T1-weighted images, 12 patients exhibited isointensity $(70.6 \%)$, and 4 exhibited hyperintensity (23.5\%). Twelve exhibited signal void on T2-weighted images (70.6\%). The loca- 


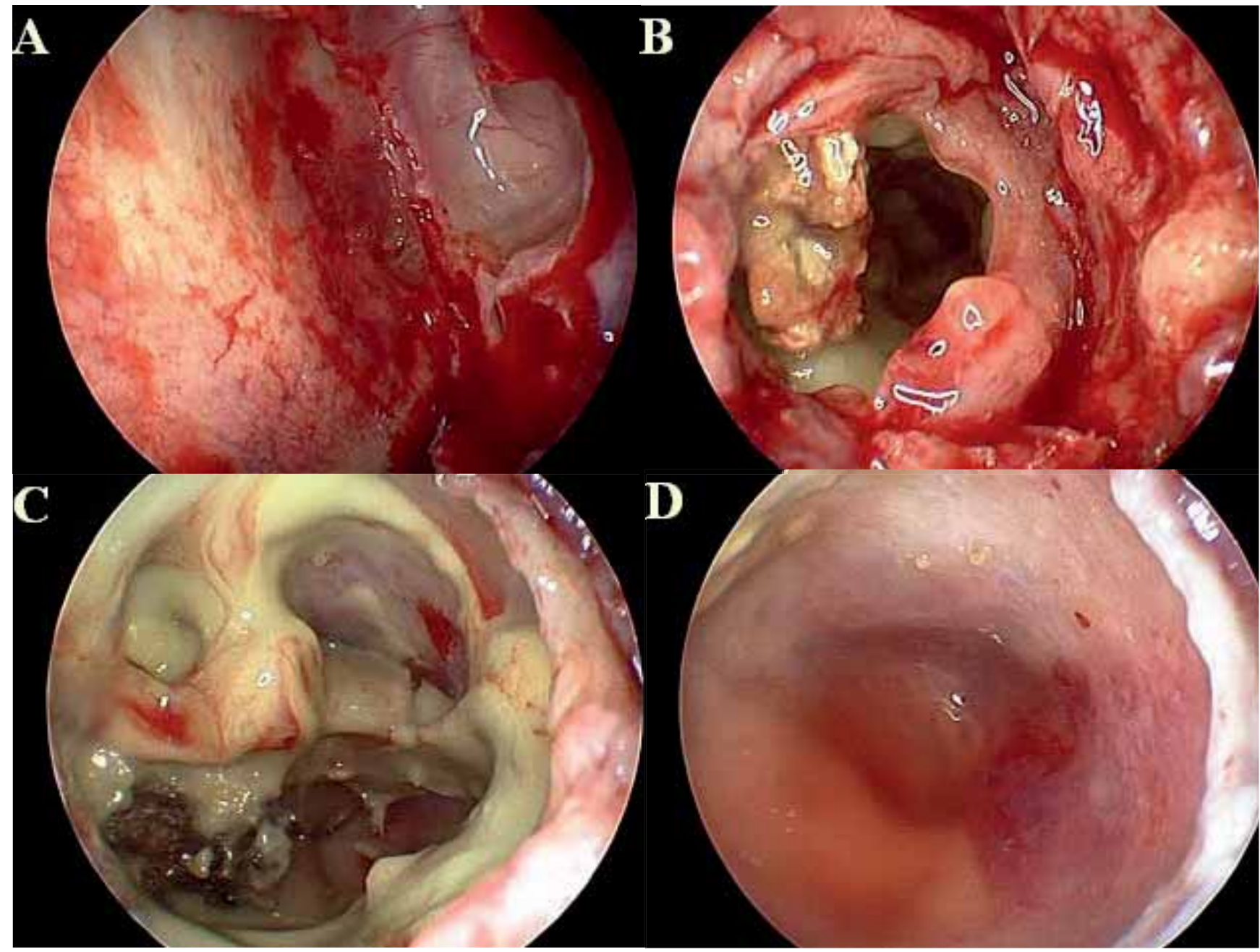

Figure 3. The surgical procedure involved in the removal of the fungus ball from the sphenoid sinus. A) Sphenoethmoidal recess after the partial resection of the superior turbinate while performing paraseptal sphenoidotomy. B) and C) A clay-like material in the sphenoid sinus. D) The end of the procedure after irrigation of the sinus with normal saline.

tions of sphenoid FB showed that the lesion was on the right side in 7 patients $(24.1 \%)$, on the left side in 21 (72.4\%), and on both sides in 1 (3.4\%)

For most patients ( 28 of $29,96.6 \%$ ), the surgical procedures were performed under local aneasthesia. For all patients, surgery was performed via the transnasal paraseptal approach. All patients exhibited clay-like material suggestive of fungal infection during the operation. Histological examination confirmed aspergillomas in all patients. No patient was treated with systemic antifungal agents. Twenty-one of the 23 symptomatic patients showed improvement in their condition immediately after the operation. The mean period of follow-up was 9.4 months (0-64 months). Recurrence was not observed in any patients.

\section{Discussion}

Non-invasive fungal rhinosinusitis can be divided into 3 subtypes: 1) saprophytic fungal infection, 2) FB, and 3) eosinophil- related fungal rhinosinusitis, including allergic fungal rhinosinusitis ${ }^{(13)}$. The sinus in which FB is involved most commonly, is the maxillary $(82.7 \%-94.0 \%)$, followed by the sphenoid sinus $(4.0 \%-14.4 \%)^{(5,6,8-11)}$. The frequency of involvement of the sphenoid sinus was $8 \%$ in our study, similar to the rates reported in the literature.

FB has been reported to occur predominantly in middleaged and elderly women ${ }^{(5-8,10,11,13,14)}$. In our study as well as other studies involving isolated sphenoid $F B^{(9,15-17)}$, the progress of $\mathrm{FB}$ was similar to that observed in other paranasal sinuses. While the definite reason for a female predominance has not been fully explained, several studies have suggested that hormonal factors ${ }^{(6,8,14)}$ and longer life expectancy of women ${ }^{(18)}$ may be relevant.

The symptoms of isolated sphenoid FB and ISSD are vague and difficult to characterise; however, headache is reportedly the most common symptom for both ${ }^{(1-3,9,10,15-17,19)}$. 
The localization of the headaches was not precisely defined in other studies associated with isolated sphenoid FB or ISSD, presumably because the localization of headaches is not necessarily associated with the affected paranasal sinus. Although the localization of headaches has been variously described in the literature, the most common regions involved are the frontal, retro-orbital, and occipital. In our patients, the most common symptom was headache (69\%), and the distribution of the headaches was diverse; frontal (35\%) was followed by parietal (25\%), whole (25\%), occipital (10\%), and vertex (5\%). Lee et al. ${ }^{(16)}$ have reported that headaches in the peri-orbital or retro-orbital regions were correlated with isolated sphenoid FB. No patients presented with headache in the peri-orbital or retro-orbital regions in our study, and retro-orbital headache accounted for $16.1 \%$ in a study by Pagella et al. ${ }^{(15)}$. Based on these considerations, retro-orbital headache cannot be significantly associated with isolated sphenoid FB. The predominant region of head aches in isolated sphenoid FB was found to be nonspecific and subtle in this study.

In this study, symptoms other than headaches were rarely presented in contrast with other ISSD or isolated sphenoid FB studies ${ }^{(1-3,15-17,19)}$. In the literature, visual disturbance is reported to be a common symptom, with incidence ranging from $14 \%$ to $52 \%$ in ISSD and isolated sphenoid FB ${ }^{(1-3,15,16,20)}$. The pathophysiology responsible for visual problems is divided into 3 categories $^{(20):}$ 1) cranial neuritis, 2) pressure ischemia, and 3) ischemic infarction. In our study, visual problems were only found in 1 patient (3.4\%); a low rate in contrast with other studies. Evidently, the patient who reported visual disturbance in our study had recovered completely due to the endoscopic sinus surgery, which was performed less than 1 month after symptom development. This is concordant with Lee et al. ${ }^{(20)}$, who reported that visual disturbances lasting less than 6 months may get restored after the operation.

Nasal symptoms, including posterior nasal drip, nasal obstruction, and mucopurulent rhinorrhea, presented at a low rate in our study in contrast with other studies ${ }^{(1-3,15-17,19)}$. Lee et al. ${ }^{(16)}$ reported that posterior nasal drip was one of the characteristics of isolated sphenoid FB. Of our patients, only 2 complained of posterior nasal drip (6.9\%); thus, it did not seem to be strongly associated with isolated sphenoid FB. Epistaxis is reported to develop frequently in patients with isolated sphenoid FB (1). However, no patient reported epistaxis in this study. The most common findings of the endoscopic examination of isolated sphenoid FB are reportedly 'nonspecific', followed by purulent discharge ${ }^{(15-17)}$. Other studies have reported that the sphenoethmoidal recess showed inflammatory mucosal changes in most cases, with or without purulent discharge or po- lyps ${ }^{(10)}$. Martin et al. ${ }^{(3)}$ reported that all 3 of the isolated sphenoid FB patients they investigated exhibited purulent discharge. However, our most common endoscopic finding was 'nonspecific', (75.9\%) which is in accordance with other studies. The second most common finding was polyp (13.8\%) in the sphenoethmoidal recess. We also concluded that it was impossible to exclude isolated sphenoid FB on the basis of endoscopic findings alone.

On CT scans, FBs either appear as sclerosis of the sinus wall or as calcifications. The calcification, observed in radiology scans, is related to the presence of iron, manganese, and calcification in the FB. Observed frequencies of calcification in the sphenoid sinus have been reported to range from $40 \%$ to $100 \%(6,9,11,16,17)$. We observed calcification within this range (69.6\%). In contrast with other studies $(9,11,17)$, sclerosis of the sinus wall was observed at a high frequency (95.6\%). Although the pathophysiology of sclerosis is not well understood, Bowman et al. ${ }^{(17)}$ proposed that it may be a chronic inflammatory response. We concluded that the radiological features of isolated sphenoid FB are associated with sclerosis of the sinus wall rather than calcification on the $\mathrm{CT}$ scans. In our study, the FB frequently occurred at the larger sinus (60.9\%). Bowman et al. ${ }^{(17)}$ have speculated that the cause of involvement of the larger sinus is expansion of the fungal disease.

MRI is not essential, except in cases where bony erosion is evident or the FB is connected with surrounding structures such as orbital content or brain structures observed via CT scans. The results of MRI may often be inconclusive. However, the radiological features observed on $\mathrm{MRI}$ scans of $\mathrm{FB}$ are known to show signal void in T2-weighted images $(6,9,10,17)$. We observed signal void in $70.6 \%$ of T2-weighted images. The occurrence of signal void has been attributed to the magnetic susceptibility of calcification, iron, and manganese. MRI is used to differentiate a FB from a mucocoele, but it is difficult to distinguish between FB and air in the sinus. Therefore, we concluded that MRI was not sufficient to diagnose FB. Leroux et al. (9) have recommended that MRI be used to rule out other causes of headache or to evaluate complications.

The approaches to sphenoid sinus are largely divided into transnasal endoscopic (including paraseptal and transethmoidal) and transseptal approaches. Many authors have frequently utilised a transnasal endoscopic approach, since the year $2000^{(1-3,15-17)}$. All patients underwent transnasal paraseptal sphenoidotomy in this study. This technique facilitated excellent visualization and proved to be a safe approach, providing direct access to the sphenoid sinus. Kieff et al. ${ }^{(19)}$ and Kim et al. ${ }^{(2)}$ have reported that transnasal paraseptal endoscopic sphenoidotomy is a regionally limited and minimally disruptive technique and therefore is 
not only fast with regard to surgery and healing time, but also complications rarely develop. No significant intraoperative or postoperative complications were observed in our study. The recurrence of isolated sphenoid FB is uncommon ${ }^{(9,15,17)}$, and no recurrence was observed in our study. In the literature, it has been suggested that the patency of the sphenoidal ostium is important to prevent isolated sphenoid FB from recurring after sphenoidotomy ${ }^{(7,17)}$. Although 2 patients developed narrowing at the ostium of the sphenoid sinus, the recurrence of $F B$ was not observed. We think that the recurrence of $F B$ is associated with remnant fungal debris rather than closure of the ostium. Therefore, we recommend performing sinus irrigation with saline solution to completely clear any remnant fungal debris and facilitate rehabilitation of the sinus. Chobillon et al. ${ }^{(14)}$ have suggested that the recurrence of FB is related to residual fungal debris in the sinus after surgery. In the literature, it is recommended that lavage of the sinus be performed with normal saline or iodine solution to remove any remnant fungal debris ${ }^{(6,7,14,15)}$. Although the follow-up period was short compared to some studies in the literature $(9,15,17)$, no recurrence was observed in any patient. Most of the surgical procedures were performed under general anaesthesia in other studies ${ }^{(15,19,21)}$, but all, except 1 patient, underwent endoscopic sphenoidotomy under local anaesthesia in our study. Endoscopic sinus surgery using local anaesthesia is known to result in shorter total operative and recovery times than that employing general anaesthesia ${ }^{(21)}$. If the patients consented and the FB was not connected with adjacent structures, we reasoned that performing sphenoidotomy under local anaesthetic would be sufficient. Based on the literature, FB does not require treatment with antifungal agents postoperatively if there is no evidence of mucosal invasion in the sinus ${ }^{(10,15)}$. If the patient has multiple nerve palsies (e.g., impaired visual acuity and ocular movement) or is immunocompromised, invasive fungal rhinosinusitis should be suspected in the absence of mucosal invasion on histopathological examinations. In such cases, we recommend that patients use preventive antifungal agents. Toussain et al. ${ }^{(11)}$ have recommended that if immunocompromised patients show clinical signs of invasion without histopathological signs of invasion, they should use antifungal agents postoperatively.

In summary, isolated sphenoid FB may go undiagnosed because it is an uncommon disease of the paranasal sinus, and the symptoms are usually nonspecific. In this study, FB was observed to predominantly occur in older women and was characterised by headaches and sclerosis of the sinus wall observed on CT scans. The localization of the headache was variable among the cases, and thus, the region of the headache is conceivably not useful when suspecting isolated sphenoid FB. Intractable headache without other neurological complications could be suggestive of isolated sphenoid FB. We recommend that either CT or MRI be performed to differentiate it from other diseases. If radiological findings do not indicate involvement of adjacent structures with the sphenoid sinus, endoscopic transnasal paraseptal sphenoidotomy was performed for the removal of the FB under local anaesthesia. Performing this technique under local anaesthesia may facilitate rapid recovery and may result in a low morbidity rate.

\section{Acknowledgement}

None

\section{Authorship contribution}

Conception and design: THK, JSK

Provision of study material or patients: THK, SJH

Collection/assembly of data: KJN, JHP

Data analysis and interpretation: THK

Manuscript writing: THK

Final approval of the manuscript:THK, JSK

\section{Conflict of interest}

The authors do not have any conflicts of interest to declare.

\section{References}

1. Wang ZM, Kanoh N, Dai CF, et al. Isolated sphenoid sinus disease: an analysis of 122 cases. Ann Otol Rhinol Laryngol. 2002; 111: 323-327.

2. Kim SW, Kim DW, Kong IG, et al. Isolated sphenoid sinus diseases: report of 76 cases. Acta Otolaryngol. 2008; 128: 455-459.

3. Martin TJ, Smith TL, Smith MM, Loehrl TA. Evaluation and surgical management of isolated sphenoid sinus disease. Arch Otolaryngol Head Neck Surg. 2002; 128: 1413-1419.

4. $\mathrm{Ng} \mathrm{YH}$, Sethi DS. Isolated sphenoid sinus disease: differential diagnosis and management. Curr Opin Otolaryngol Head Neck
Surg. 2011; 19: 16-20.

5. Dufour X, Kauffmann-Lacroix C, Ferrie JC, et al. Paranasal sinus fungus ball and surgery: a review of 175 cases. Rhinology. 2005; 43: 34-39.

6. Nicolai , Lombardi $D$, Tomenzoli $D$, et al. Fungus ball of the paranasal sinuses: experience in 160 patients treated with endoscopic surgery. Laryngoscope. 2009; 119 : 2275-2279.

7. Pagella F, Matti E, De Bernardi F, et al. Paranasal sinus fungus ball: diagnosis and management. Mycoses. 2007; 50: 451-456.

8. Dufour X, Kauffmann-Lacroix C, Ferrie JC, Goujon JM, Rodier MH, Klossek JM. Paranasal sinus fungus ball: epidemiology, clinical features and diagnosis. A retrospective analysis of 173 cases from a single medical center in France, 1989-2002. Med Mycol. 2006; 44: 61-67.

9. Leroux E, Valade D, Guichard JP, Herman P. Sphenoid fungus balls: clinical presentation and long-term follow-up in 24 patients. Cephalalgia. 2009; 29: 1218-1223.

10. Grosjean P, Weber R. Fungus balls of the paranasal sinuses: a review. Eur Arch Otorhinolaryngol. 2007; 264: 461-470.

11. Toussain G, Botterel F, Alsamad IA, et al. Sinus fungal balls: characteristics and management in patients with host factors for invasive infection. Rhinology. 2012; 50: 269276. 
12. deShazo RD, O'Brien M, Chapin K, et al. Criteria for the diagnosis of sinus mycetoma. J Allergy Clin Immunol. 1997; 99: 475485.

13. Chakrabarti A, Denning DW, Ferguson BJ, et al. Fungal rhinosinusitis: a categorization and definitional schema addressing current controversies. Laryngoscope. 2009; 119: 1809-1818.

14. Chobillon MA, Jankowski R. What are the advantages of the endoscopic canine fossa approach in treating maxillary sinus aspergillomas? Rhinology. 2004; 42: 230-235.

15. Pagella F, Pusateri A, Matti E, et al. Sphenoid sinus fungus ball: our experience. Am Rhinol Allergy. 2011; 25: 276-280.

16. Lee TJ, Huang SF, Chang PH. Characteristics of isolated sphenoid sinus aspergilloma: report of twelve cases and literature review. Ann Otol Rhinol Laryngol. 2009; 118: 211 -
217

17. Bowman J, Panizza B, Gandhi M. Sphenoid sinus fungal balls. Ann Otol Rhinol Laryngol 2007; 116: 514-519.

18. Ferguson BJ. Fungus balls of the paranasal sinuses. Otolaryngol Clin North Am. 2000; 33: 389-398.

19. Kieff DA, Busaba N. Treatment of isolated sphenoid sinus inflammatory disease by endoscopic sphenoidotomy without ethmoidectomy. Laryngoscope. 2002; 112 2186-2188.

20. Lee LA, Huang CC, Lee TJ. Prolonged visual disturbance secondary to isolated sphenoid sinus disease. Laryngoscope. 2004 114: 986-990.

21. Fedok FG, Ferraro RE, Kingsley CP, Fornadley JA. Operative times, postanesthesia recovery times, and complications during sinonasal surgery using general anesthesia and local anesthesia with sedation. Otolaryngol Head Neck Surg. 2000; 122: 560-566.

Jung Soo Kim

Department of Otorhinolaryngology-

Head \& Neck Surgery,

Kyungpook National University 130 Dongdeok-ro, Jung-gu, Daegu 700-721, South Korea

Tel: +82-53-200-5777

Fax: +82-53-423-4524

E-mail: sookim@knu.ac.kr

\section{1th INTERNATIONAL COURSE IN ADVANCED SINUS SURGERY TECHNIQUES}

Dissection course with fresh frozen cadaver heads

Teacher of Honour:

Robert C Kern, MD

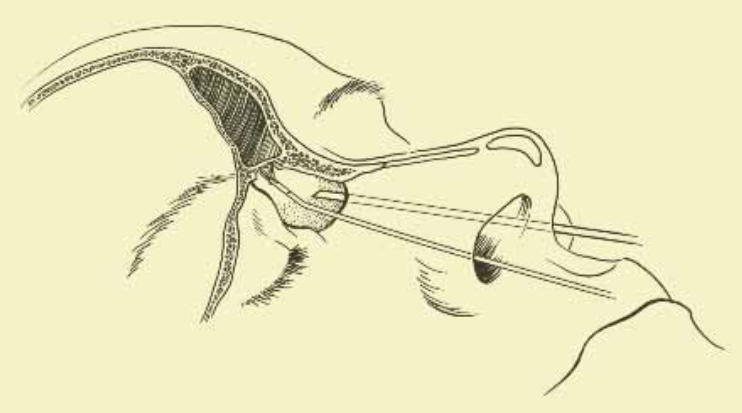

March 27-28, 2014

Department of Otorhinolaryngology Academic Medical Center of the University of Amsterdam

The Netherlands

For further information contact Wytske I. Fokkens, MD, PhD ENT dept. AMC Course Secretariat Tel: 0031205668586 / Fax 0031205669573

Email:m.b.vanhuiden@amc.uva.nl Web: www sinuscourse $n l$

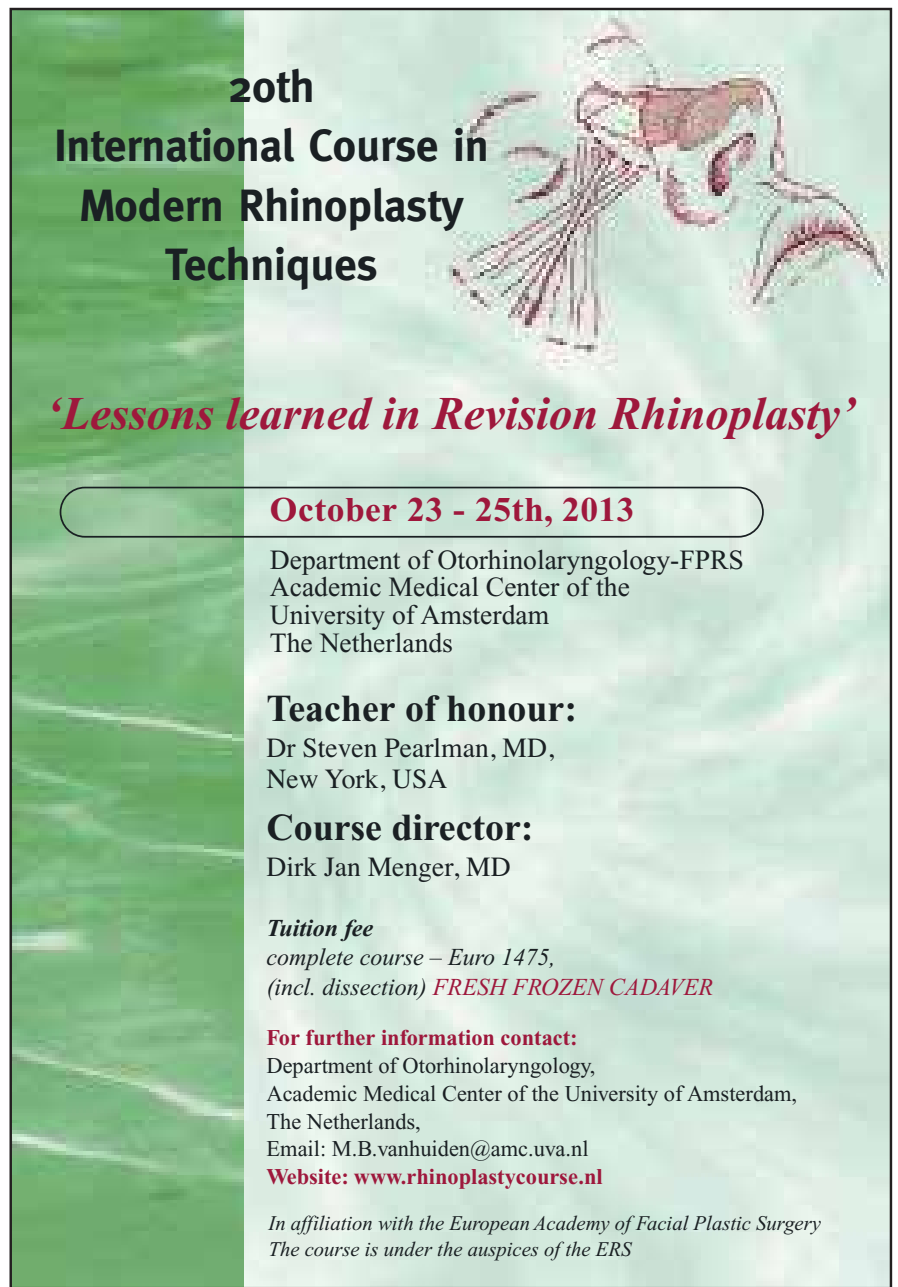

